\title{
Alice's Adventures in an Immersive Mixed Reality Environment
}

\author{
Marija Nakevska, Jun Hu, Geert Langereis and Matthias Rauterberg* \\ Department of Industrial Design, Eindhoven University of Technology
}

\begin{abstract}
Immersive mixed reality environments offer new possibilities to reproduce reality or embodied presence with constructing elaborate fantasy worlds and provide to the user an intense and seemingly real experience. Mixed reality gives possibilities to create deeply dimensional narratives and simulations that put the user in the center of the action. In this work we describe an interactive mixed reality installation named Alice, consisting of six separate stages based on the narrative 'Alice's Adventures in Wonderland'. To be able to achieve the intended experience we have to build complex and heterogeneous distributed system, composed of sensors, actuators, virtual reality, application components and variety of processing components that manage the flow of context information between the sensors/actuators and applications.
\end{abstract}

Keywords: Immersive mixed reality, cultural computing.

\section{INTRODUCTION}

Immersive mixed reality environments are using a large palette of techniques to create fantasy worlds, including various multisensory effects like various forms of tactile sensations, fog, movement of a seat or floor, and artificially produced smells. All the components of other engaging forms of digital entertainment can be included; as sound and moving images, computergenerated intelligent characters, three-dimensional simulations, embodied characters and new types of spaces.

We are exploring a new application of mixed reality for a novel direction in human-computer interaction named 'cultural computing'. Tosa et al. are using mixed reality technology to engage the users to the certain elements from Eastern, Japanese Zen culture [1,2]. Within the ALICE project we aim to create an interactive installation based on the narrative of 'Alice's Adventures in Wonderland' that encourages people in Western culture to reflect on themselves, addressing issues such as logic, rationality, and self [3]. The design includes implementation of a distributed system consisting of autonomous components, in a range from high-performance mainframe computers to small sensor nodes. We have to take in consideration both formal and spatial elements of the system and interprocess communication and synchronization. Next, we are presenting the ALICE project with the challenges of the hardware and software design to provide the intended experience.

\section{The ALICE PROJECT}

The ALICE installation consists of six consecutive stages based on the narrative 'Alice's Adventures in Wonderland'; each represents a chapter or part of it. The installation is built on two floors (the ground floor represented in Figure 1), and the designed

\footnotetext{
* \{m.nakevska,j.hu,g.r.langereis,g.w.m.rauterberg\}@tue.nl
}

IEEE International Symposium on Mixed and Augmented Reality 2012 Science and Technology Proceedings 5 - 8 November 2012, Atlanta, Georgia 978-1-4673-4662-7/12/\$31.00 @2012 IEEE experience is for one person at a time. From start to end the user undergoes immersion that engages the user in an experience closer to the author of the book has described for the main character Alice.

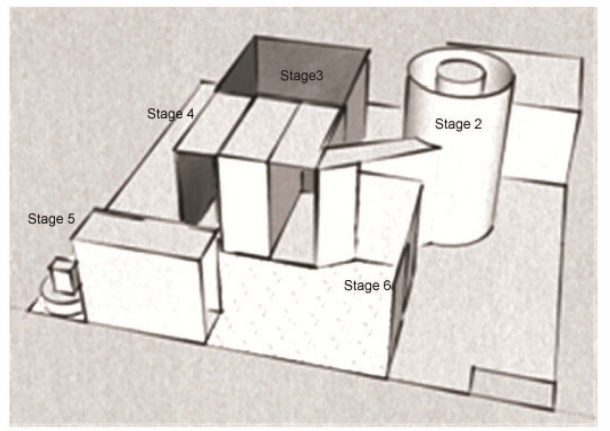

Figure 1: 3D model of the actual installation, presenting Stage 2, 3, 4,5 and 6.

Following, we will explain the function of each part, summarizing the section from the original narrative, impression of the design of each stage and its technical implementation:

Stage-1: 'In the park'; In the first scene of Carroll's original book, Alice is bored and sleepy sitting on a bank with her sister. Her curiosity is triggered by white rabbit which runs, looking at its pocket watch and cries out "Oh dear! Oh Dear! I shall be late!". We simulate natural environment in which the user is expected to wait until he/she is bored and thereby enters a similar state of mind as Alice in the original story. The visitor is surrounded with $360^{\circ}$ panorama picture, supported with strong light behind the printed fabric which gives impression of brightness of a summer day. The visual simulation is supported by two virtual reality projections, also supported with appropriate audio simulation of natural environment. The appearance of the rabbit in the scene has a goal to seduce the visitor to follow with a catch-me-if-you-can behavior. To imitate the movement of the rabbit we are using virtual reality projection and physical actuation of artificial grass, successively synchronized.

Stage-2: 'Down the Rabbit Hole'; Alice falls down the rabbit hole in an unusual fashion; the fall takes such a long time that Alice engages in a conversation with herself. In the ALICE installation we are bridging the fall in the rabbit hole with a seat mounted on a rail. Once the visitor is safely seated, controlled gate is open and it takes the visitor down the tunnel in a spiral movement (see Figure 2 ). The speed of the fall is very slow and the visitor is able to interact with objects that are attached to the walls of the rabbit hole, such as cupboards and bookshelves.

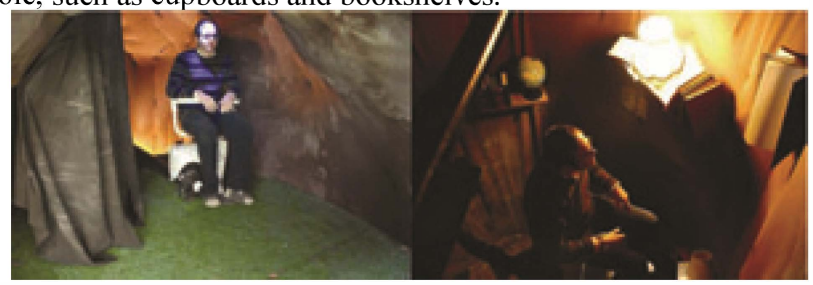

Figure 2: Visitor entering the rabbit hole. 
Stage-3: 'Shrinking and Growing'; Alice enters a dark corridor with many doors, which are all locked. A tiny door leads to a garden, but Alice is too tall to enter; she notices a bottle labeled 'Drink Me' and later a little cake labeled 'Eat Me'. By drinking from the bottle, she shrinks and by eating the cake, she grows. Eventually, she manages to have the appropriate size to enter through the tiny door.

To be able to manipulate the relative size in comparison to the environment we are using a cubic CAVE. The walls and the ceiling are made of white semi-transparent material. We used the back-projection method to project a seamless virtual environment onto the walls of the CAVE. (see Figure 3).

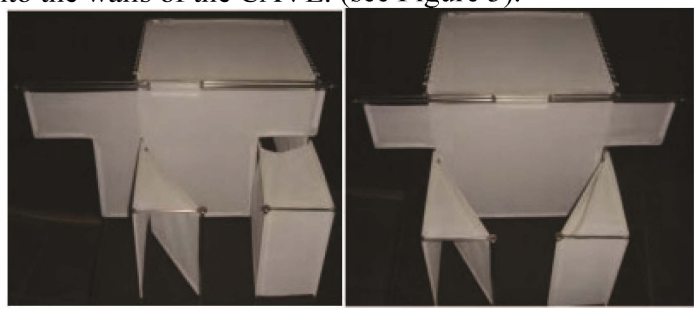

Figure 3: Model of the physical implementation of the 5-sided CAVE with the sliding doors.

The visitor entered the cave and had the impression to stand in a virtual room (Figure 4(a)). A cookies box labeled 'Eat Me' and a bottle labeled 'Drink Me' are placed on top of a small table (see Figure 4(b)). When the visitor drinks from the bottle, the virtual room enlarges, giving the impression that the visitor is shrinking. When eating the cookie, the virtual room shrinks, giving the visitor the impression that he/she is growing. On one side of the room, a door is shown. Once the visitor reaches the appropriate size, the wall on which the door is shown moves aside and thereby allows the visitor to enter stage 4 .
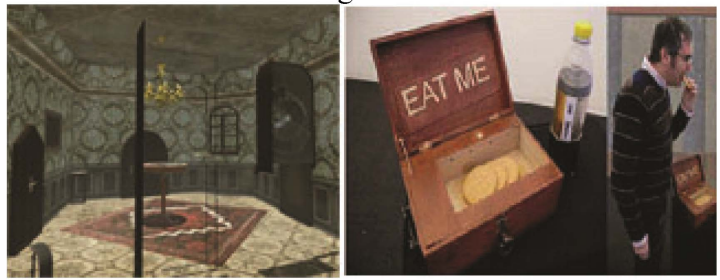

Figure 4: (a)Virtual reality model of the projected room and (b) "Eat me, drink me" interaction equipped with sensors.

We used CryEngine 3 to synchronize the five projectors. The model of the virtual room was developed in Maya. The bottle features touch and tilt sensors to detect drinking. The cookie box is equipped with a light sensor to detect when the user takes a cookie and microphone that allows us to detect the visitor's chewing sounds when eating the cookie.

Stage-4: "Pool of tears"; During her growing and shrinking experience, Alice cries many tears. She talks to a mouse that swims alongside her and together with some other animal they finally reach the shore. When the visitor enters stage 4 , the sea with a mouse in it is projected on one of the walls. In addition, a smoke machine creates an impression of moisture and adds a mystical feeling to this stage. The visitor walks along the projection and thereby enters stage 5 . We created a virtual pool of tears with the use of back-projection and we also use fog machine to enhance the effect (Figure 5(a)). The animation of the mouse in the water was created in 3D Studio Max.

Stage-5: Advice from a Caterpillar'; The state of confusion in the Alice character is emphasized in her conversation with the Caterpillar: 'Who are you?'. We created a robotic caterpillar to engage the visitor in a similar dialogue (Figure 5(b)).
Microphones recorded the utterances of the visitor and a simple dialogue system manages the conversation. Since most of the questions are metaphysical or mystical, a conclusive dialogue can be created without an extensive Artificial Intelligence for the caterpillar.
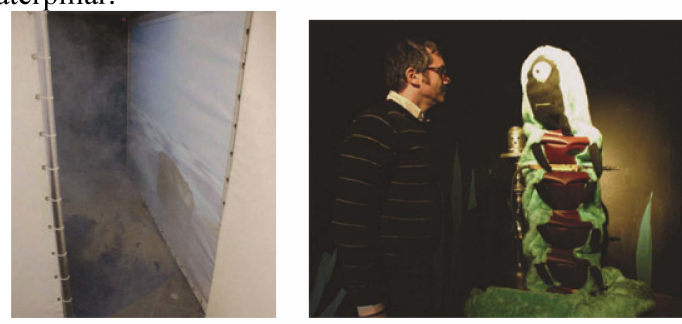

Figure 5: (a) Pool of tears- projection and effect produced with smoke machine (b) A visitor is talking with the caterpillar, a robotic dialogue agent.

Stage-6: 'Talk with the Cheshire Cat'; The Cheshire cat involves Alice in a dialogue about logical reasoning and madness. During the dialogue the cat disappears at times completely and sometimes only its grin remains visible. We created a virtual Cheshire cat that is projected on a screen (Figure 6). When the visitor approaches the cat, it engages him/her in dialogue similar to the one in Carroll's book.
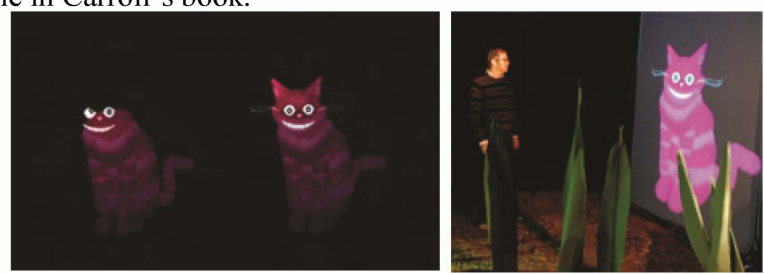

Figure 6: Left, different transformation of the Chashire cat, right visitor engaged in conversation.

A Microphone was hidden next to the screen to record the visitor's responses. A simple 2D animation tool (Adobe Director) is used for the animation of the cat and the dialogue management. Similar to the Caterpillar, most of the questions are metaphysical or mystical, and hence a conclusive dialogue can be created without an extensive Artificial Intelligence.

\section{CONCLUSION}

In the ALICE project we took advantage of novel technologies and mixed reality, to create rich experiences that influence the visitor. We are constructing in real-time a personalized narrative and flow of events. To maximize the potential for engaging the user in an interaction, we create immersive and interactive environment that reacts to his/her input and behaviors including motions, displacements and physical environments. In order to obtain a consistent global behavior the system has to provide support for the interactions between entities, coordination between sensing data, pre-defined scenarios and selection of actions by the autonomous environment.

\section{REFERENCES}

[1] N. Tosa, S. Matsuoka and H. Thomas. Inter-culture Computing: ZENetic Computer. In ACM SIGGRAPH 2004 Emerging technologies 2004.

[2] N.Tosa, S. Matsuoka, B. Ellis, H. Ueda \& R. Nakatsu. Cultural Computing with Context Aware Application: ZENetic Computer. Lecture Notes in Computer Science, vol. 3711, pp. 13 - 23.

[3] M. Rauterberg. From Personal to Cultural Computing: How to Assess A Cultural Experience. In uDayIV-Information nutzbar machen, 2006, Pabst. 